\title{
Neonatal alloimmune thrombocytopenia
}

This article was published in the following Dove Press journal:

International Journal of Clinical Transfusion Medicine

8 June 2015

Number of times this article has been viewed

\section{Maria Teresa Mella \\ Keith A Eddleman}

Department of Obstetrics and Gynecology, Division of Maternal Fetal Medicine, Mount Sinai School of Medicine, New York, NY, USA
Correspondence: Maria Teresa Mella Department of Obstetrics and Gynecology, Division of Maternal Fetal Medicine, Mount Sinai School of Medicine, 5 East 98th Street, 2nd Floor, New York, NY 10029, USA Email mariateresa.mella@mssm.edu
Abstract: Neonatal alloimmune thrombocytopenia occurs in one in 1,000-1,500 live births and is the most common cause of severe thrombocytopenia and intracranial hemorrhage in term infants. It is the equivalent of red blood cell alloimmunization and is due to transplacental passage of maternal antibodies against paternally derived fetal platelet antigens. A diagnosis of neonatal alloimmune thrombocytopenia should be considered for any neonate with unexplained thrombocytopenia. Once the diagnosis is made, it is known that all subsequent pregnancies are at risk for severe disease. In order to prevent the devastating and potentially life-threatening manifestations of the disease, the goal is to initiate treatment early with serial percutaneous umbilical blood sampling, intravenous immunoglobulin administration, prednisone, and/or fetal platelet transfusions. Timing of delivery is variable with delivery for severe disease recommended at an earlier gestational age. Vaginal delivery can be considered if the fetal platelet count is greater than 50,000-100,000 $\mu \mathrm{L}$. Thrombocytopenia due to neonatal alloimmune thrombocytopenia usually resolves spontaneously within 1-2 weeks after delivery, but a platelet transfusion may be necessary to prevent a serious hemorrhagic event. In all cases, a multidisciplinary approach to care should be undertaken with delivery at a tertiary care center.

Keywords: neonatal thrombocytopenia, fetal therapy, intracranial hemorrhage, intravenous immunoglobulin, alloimmunization

\section{Background}

Neonatal alloimmune thrombocytopenia (NAIT) is defined as a fetal/neonatal platelet count of less than $150,000 / \mu \mathrm{L}$ resulting from maternal sensitization to incompatible fetal platelet antigens. Although it is a rare condition, it is the most common cause of severe thrombocytopenia (platelet count less than $50,000 / \mu \mathrm{L}$ ), in the fetal and neonatal period and the most common cause of intracranial hemorrhage $(\mathrm{ICH})$ in term infants. The reported incidence of NAIT is one in 1,000-1,500 live births. ${ }^{1}$ ICH occurs in 10 per 100,000 cases, with most cases occurring before 28 weeks. ${ }^{2}$ NAIT tends to be more severe with each subsequent pregnancy and typically occurs at an earlier gestational age than the first affected pregnancy. ${ }^{3}$ Therefore, it is important to consider this diagnosis in all cases of neonatal thrombocytopenia in order to determine future pregnancy risk and an acceptable treatment plan. This article is a review of NAIT including the pathogenesis of NAIT as well as the antenatal and postnatal treatment strategies currently available.

\section{Pathogenesis}

NAIT is the platelet equivalent of red blood cell alloimmunization. Fetal platelets contain paternally derived platelet antigens that maternal platelets lack. After an episode 
of fetomaternal hemorrhage, maternal immunoglobulin $\mathrm{G}$ (IgG) antibodies are created against these specific antigens, cross the placenta, and bind to fetal platelet antigens resulting in sequestration and destruction of fetal platelets. This process occurs in the fetal reticulo-endothelial system and can also lead to impaired platelet aggregation. This results in fetal/neonatal thrombocytopenia in varying degrees of severity. Fetomaternal hemorrhage occurs most often at delivery. Cesarean delivery, multifetal gestation, bleeding placenta previa or abruption, manual removal of the placenta, and intrauterine manipulation are the most common causes that increase the volume of fetomaternal hemorrhage. Other causes of fetomaternal hemorrhage include any antepartum vaginal bleeding after spontaneous, threatened, or induced abortions; trauma; external cephalic version; or after invasive procedures such as chorionic villus sampling, amniocentesis, or fetal blood sampling (FBS). Unlike Rhesus (Rh) alloimmunization, $40 \%-60 \%$ of index pregnancies can be affected by NAIT. This is due to the transplacental transport and coating of fetal platelets with maternal IgG antiplatelet antibodies to glycoproteins on the fetal platelet antigens. ${ }^{4}$

Fetal human platelet antigens (HPAs) are located on cell membrane glycoproteins and are inherited in an autosomal co-dominant fashion. To date, 24 HPAs have been described and are grouped in one of five major bi-allelic systems of platelet-specific antigens (each differs by a single amino acid): HPA-1 through HPA-5. ${ }^{5}$ Within this nomenclature, the platelet alloantigens are further defined according to the frequency in which they appear within the population, with "a" being a high-frequency alloantigen and " $b$ " a lowfrequency alloantigen (Table 1). ${ }^{6,7}$ The most common HPA haplotype is HPA-1a. Approximately $80 \%$ of pregnancies affected by NAIT have maternal antibodies that are directed against platelet antigen HPA-1a with the remaining 20\% being affected by the other HPA types. Studies have shown that approximately $98 \%$ of Caucasian women express HPA-1a (genotype HPA-1a/HPA-1a or HPA1a/HPA1b) and about $2 \%$ of Caucasian women are HPA-1 a negative (genotype HPA-1b/

Table I Human platelet alloantigens

\begin{tabular}{|c|c|c|c|c|}
\hline Antigen & Other names & Glycoprotein location & Amino acid change & Phenotype frequency \\
\hline HPA-Ia & $\mathrm{PI}^{\mathrm{A}}, \mathrm{ZW}$ & GPIIla & Leu $<$ - $>$ Pro33 & $72 \% \mathrm{a} / \mathrm{a}$ \\
\hline \multirow[t]{2}{*}{ HPA-Ib } & & & & $26 \% \mathrm{a} / \mathrm{b}$ \\
\hline & & & & $2 \% \mathrm{~b} / \mathrm{b}$ \\
\hline HPA-2a & Ko, Sib & GPIb & Thr $<->$ Met $\mid 45$ & $85 \% \mathrm{a} / \mathrm{a}$ \\
\hline \multirow[t]{2}{*}{ HPA-2b } & & & & $14 \% \mathrm{a} / \mathrm{b}$ \\
\hline & & & & $\mathrm{l} \% \mathrm{~b} / \mathrm{b}$ \\
\hline HPA-3a & Bak, Lek & GPII & Ile:Ser843 & $37 \%$ a/a \\
\hline \multirow[t]{2}{*}{ HPA-3b } & & & & $48 \% \mathrm{a} / \mathrm{b}$ \\
\hline & & & & $15 \% \mathrm{~b} / \mathrm{b}$ \\
\hline HPA-4a & Pen, Yuk & GPIIla & Arg:Gln 43 & $>99.9 \% \mathrm{a} / \mathrm{a}$ \\
\hline \multirow[t]{2}{*}{ HPA-4b } & & & & $<0.1 \% \mathrm{a} / \mathrm{b}$ \\
\hline & & & & $<0.1 \mathrm{~b} / \mathrm{b}$ \\
\hline HPA-5a & $\mathrm{Br}, \mathrm{Hc}, \mathrm{Zav}$ & GPla & Glu:Lys505 & $80 \% \mathrm{a} / \mathrm{a}$ \\
\hline \multirow[t]{2}{*}{ HPA-5b } & & & & $19 \% \mathrm{a} / \mathrm{b}$ \\
\hline & & & & $\mathrm{l} \% \mathrm{~b} / \mathrm{b}$ \\
\hline HPA-6bw & $\mathrm{Ca}^{\mathrm{a}}, \mathrm{Tu}$ & GPIIla & Arg:Gln489 & $<\mathrm{l} \% \mathrm{~b} / \mathrm{b}$ \\
\hline HPA-7bw & Mo & GPIIla & Pro $<$ - $>$ Ala407 & $<\mathrm{l} \% \mathrm{~b} / \mathrm{b}$ \\
\hline HPA-8bw & $\mathrm{Sr}^{\mathrm{a}}$ & GPIIla & Arg:Cys636 & $<0.1 \% \mathrm{~b} / \mathrm{b}$ \\
\hline HPA-9bw & $\operatorname{Max}^{\mathrm{a}}$ & GPIIla & Val:Met837 & $<\mathrm{l} \% \mathrm{~b} / \mathrm{b}$ \\
\hline HPA-IObw & $\mathrm{La}^{\mathrm{a}}$ & GPIIla & Arg:Gln62 & $\mathrm{l} \% \mathrm{~b} / \mathrm{b}$ \\
\hline HPA-I Ibw & $\mathrm{Gro}^{\mathrm{a}}$ & GPIIla & Arg:His633 & $<0.5 \% \mathrm{~b} / \mathrm{b}$ \\
\hline HPA-I2bw & $1 y^{2}$ & GPlb & Gly:Glu 15 & $\mathrm{l} \% \mathrm{~b} / \mathrm{b}$ \\
\hline HPA-I3bw & $\mathrm{Sit}^{\mathrm{a}}$ & GPla & Met:Thr799 & $<\mathrm{l} \% \mathrm{~b} / \mathrm{b}$ \\
\hline HPA-I4bw & $O \mathrm{e}^{\mathrm{a}}$ & GPIIla & Del:Lys6II & $1 \% \mathrm{~b} / \mathrm{b}$ \\
\hline HPA-I5a & Gov & CDI09 & Tyr:Ser703 & $35 \% \mathrm{a} / \mathrm{a}$ \\
\hline \multirow[t]{2}{*}{ HPA-I5b } & & & & $42 \% \mathrm{a} / \mathrm{b}$ \\
\hline & & & & $23 \% \mathrm{~b} / \mathrm{b}$ \\
\hline HPA-16bw & Duv & GPIIla & Thr:Ilel 40 & $<1 \%$ \\
\hline NA & NAK $^{a}$ & CD36 (GPIV) & & $99.8 \%$ \\
\hline
\end{tabular}

Notes: aPhenotypic frequencies for antigens shown are for white populations only. "a" and "b" under the column Phenotype frequency refer to alleles, "a" for the high-frequency and "b" for the low-frequency alleles. Reproduced with permission from: A review of pathophysiology and current treatment for neonatal alloimmune thrombocytopenia (NAIT) and introducing the Australian NAIT registry. McQuilten ZK, Wood EM, Savoia H, et al. Aust N ZJ Obstet Gynaecol, 5 I. Copyright $@$ C 201 I John Wiley and Sons. ${ }^{64}$ Abbreviations: HPA, human platelet antigen, NA, non applicable. 
HPA-1b). The second most common platelet antigen causing NAIT in Caucasians is HPA-5b antigen, followed by HPA-1b and HPA- $15 .{ }^{8}$ In the Asian population, HPA-4 is the most frequent cause of NAIT. ${ }^{-11}$ Several other platelet antigens have been associated with NAIT by varying degrees depending on ethnicity and are very rare causes of NAIT. ${ }^{12,13}$

In at-risk pregnancies, mothers are antigen negative (most commonly HPA-1b) and fathers are either antigenpositive homozygous (genotype HPA-1a/1a) or heterozygous (genotype HPA-1a/1b). As mentioned earlier, the overall frequency of the HPA-1b/1b genotype in Caucasian mothers is $2 \%$. It has been shown, that only $10 \%$ of HPA-1a-negative mothers who are exposed to HPA-1a antigen-positive platelets during pregnancy actually become immunized (develop HPA-1a alloantibodies), and of those, only $30 \%$ of fetuses/neonates will develop thrombocytopenia with $20 \%$ of those cases being severe. ${ }^{14,15,16}$ Approximately $75 \%$ of Caucasian males are homozygous for HPA-1a and 25\% are heterozygous. Given these percentages, after the index pregnancy, $85 \%$ of couples are at risk for NAIT in subsequent pregnancies. ${ }^{17}$

Multiple theories exist behind disease severity with the presence of different maternal human leukocyte antigen (HLA) types modulating severity of disease by affecting the ability to produce HPA system antibodies. It has been shown that most alloimmunization occurs in women with the presence of HLA DRB3*0101, and, in the absence of this phenotype, alloimmunization is rare. ${ }^{6,18,19}$

Additionally, it has been postulated that disease severity is also affected by other factors, including maternal concentration of IgG alloantibodies, subclass of maternal antibodies, antigen concentration on fetal/neonatal platelets, phagocyte activity in the reticulo-endothelial system, and the ability of the fetal/neonatal bone marrow to compensate for the rapid destruction of fetal platelets. ${ }^{20}$

\section{Differential diagnosis}

The differential for fetal or neonatal thrombocytopenia is extensive and can be due to problems with increased platelet consumption or destruction, decreased production, or other mixed causes (Table 2).

\section{Clinical manifestations}

Most cases of NAIT are usually diagnosed incidentally, when shortly after birth, the first-born infant is found to have thrombocytopenia with petechiae, ecchymosis, or bleeding, or in women with a previously affected pregnancy. Additionally, spontaneous fetal or neonatal ICH can be the first marker
Table 2 Differential diagnosis of neonatal alloimmune thrombocytopenia

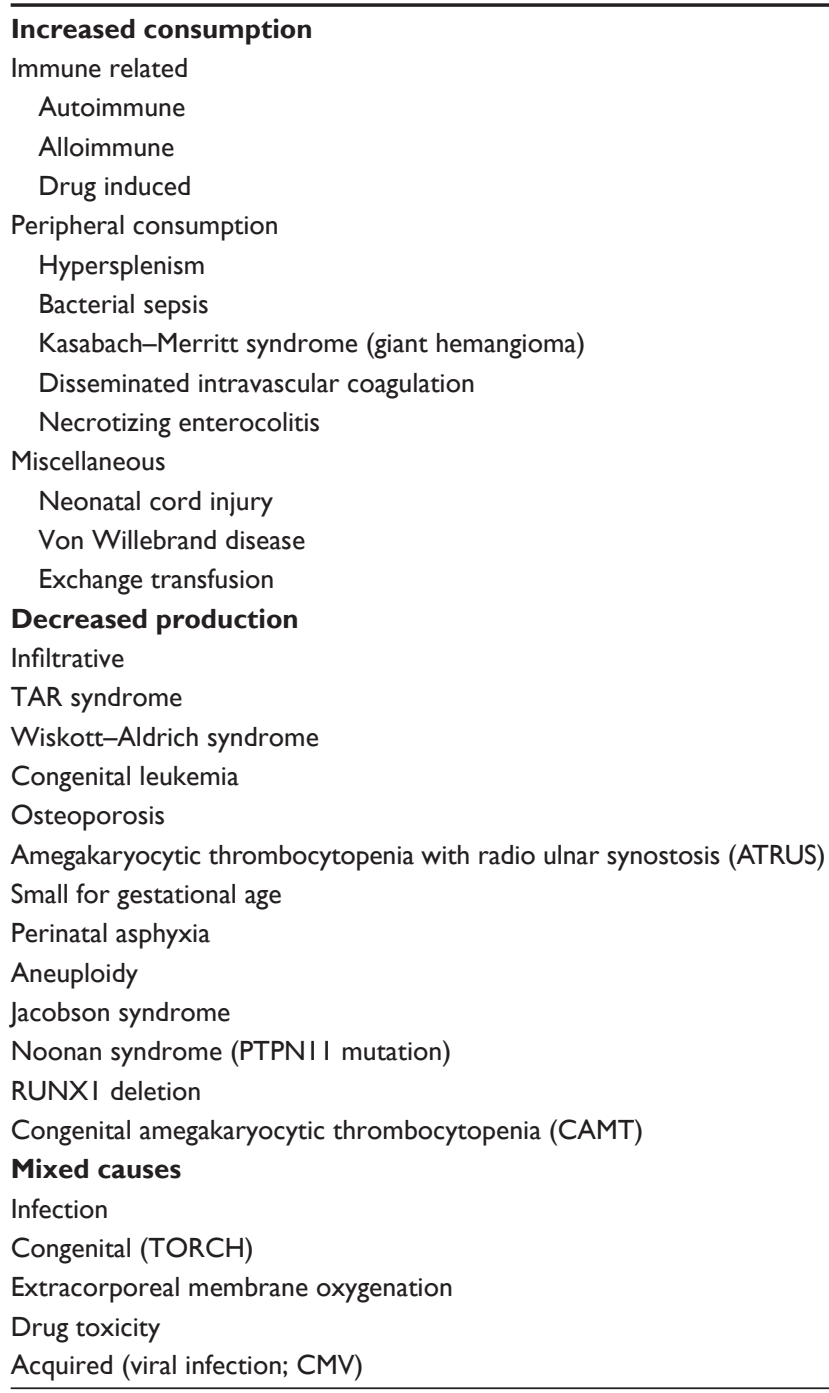

Note: Reprinted from Clin Lab Med, 33, Strong N and Eddleman KA. Diagnosis and management of neonatal alloimmune thrombocytopenia in pregnancy, 3 II-325. Copyright 2013, with permission from Elsevier. ${ }^{20}$

Abbreviations: CMV, cytomegalovirus; TAR, Thrombocytopenia Absent Radius; TORCH:Toxoplasma gondii, other viruses (HIV, measles, and so on), rubella (German measles), cytomegalovirus, and herpes simplex.

of disease. It has been shown in several studies that up to $7 \%-20 \%$ of fetuses/neonates affected by NAIT will suffer from ICH with as many as $75 \%$ of these cases occurring antenatally, diagnosed as early as the mid-second trimester. ${ }^{21,22}$

Depending on the severity of thrombocytopenia (mild, moderate, or severe), NAIT may present as a spectrum of disease findings. A platelet count of $<150,000 / \mu \mathrm{L}$ has been used to define thrombocytopenia because this is the threshold used in adults and represents the lower fifth percentile. Approximately $0.5 \%-0.9 \%$ of neonates will have thrombocytopenia, and this can be further classified as mild, moderate, or severe (Table 3). 
Table 3 Classification of neonatal thrombocytopenia with \% incidence in newborns

\begin{tabular}{lll}
\hline Thrombocytopenia & Platelet count & \% of newborns \\
\hline Mild & $100,000-150,000 / \mu \mathrm{L}$ & 0.8 \\
Moderate & $50,000-100,000 / \mu \mathrm{L}$ & 0.5 \\
Severe & $<50,000 / \mu \mathrm{L}$ & 0.2
\end{tabular}

Note: Reprinted from Clin Lab Med, 33, Strong N and Eddleman KA. Diagnosis and management of neonatal alloimmune thrombocytopenia in pregnancy, 3 I I-325. Copyright 2013, with permission from Elsevier. ${ }^{20}$

Other clinical manifestations of NAIT include gastrointestinal bleeding, hematoma, melena, hematemesis, hemoptysis, hematuria, retinal hemorrhage, or central nervous system hemorrhage. Depending on the degree of thrombocytopenia, some neonates may be asymptomatic. ${ }^{23}$

ICHs, either intraparenchymal or intraventricular hemorrhage, occur in up to $10 \%$ of pregnancies affected by NAIT. ${ }^{24,25}$ Most occur in the antepartum period; however, after delivery, the greatest risk of bleeding is in the first 96 hours of life. ${ }^{25,26}$ Intraparenchymal hemorrhages may lead to encephalomalacia with resulting porencephalic cysts. Sometimes (although less likely), intraventricular hemorrhage can result in arachnoiditis with or without hydrocephalus. Both porencephalic cysts and hydrocephalus can be diagnosed via ultrasound (Figure 1).

Long-term complications from NAIT can be seen in as many as $14 \%-26 \%$ of cases and can include mental retardation, cerebral palsy, cortical blindness, and/or seizures. ${ }^{27}$ Neonatal death can occur in up to $5 \%-10 \%$ of affected neonates. ${ }^{28-30}$ Platelets have a half-life of approximately 8-10 days, and therefore, platelet counts return to normal after 1-2 weeks at which point bleeding from NAIT no longer occurs.

Mothers of fetuses or infants with NAIT are asymptomatic with a normal platelet count and function. Factors that can predict disease severity include (+)HPA-1a antigen, alloantibody levels, and a previous child affected by an ICH. A prospective study by Killie et al found a positive correlation between maternal antibody levels and neonatal platelet counts with a $93 \%$ sensitivity in prediction of severe fetal thrombocytopenia if the anti-HPA-1a level was $>3.0 \mathrm{IU} / \mathrm{mL}$ in either week 22 or 34 of pregnancy. ${ }^{31}$ Studies have shown that the earlier the gestational age an ICH occurred in a previous pregnancy, the higher the risk for severe disease with earlier onset in each subsequent pregnancy. ${ }^{32,33}$ Studies have found that there is nearly a $100 \%$ recurrence risk of antenatal ICH in untreated pregnancies where the previous sibling was affected by an antenatal ICH. ${ }^{17,34}$
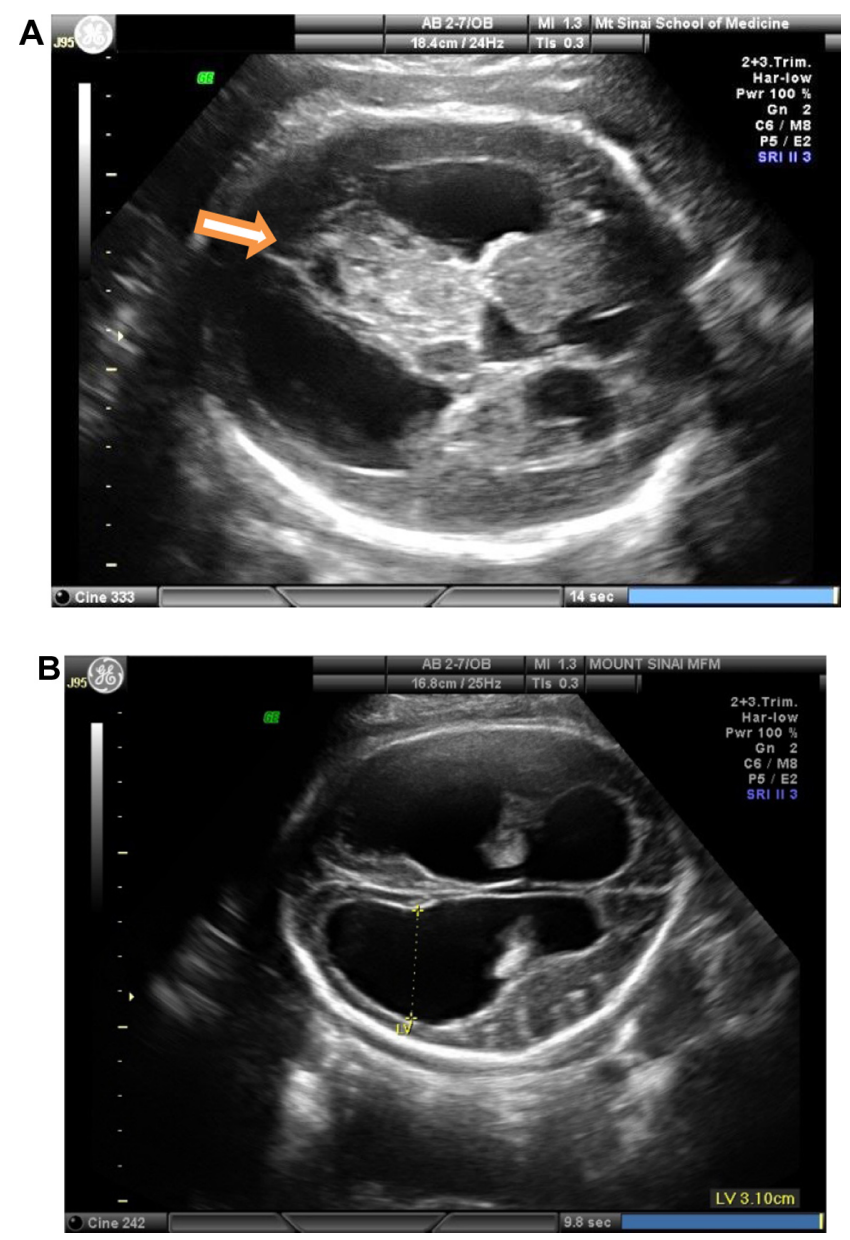

Figure I Fetal ultrasound Images of complications from neonatal alloimmune thrombocytopenia.

Notes: (A) In utero intracranial hemorrhage. This figure demonstrates an in utero organized clot that appears echogenic on ultrasound and is causing a mass effect. Arrow corresponds to area of large intracranial hemorrhage or organized clot. (B) Ventriculomegaly/hydrocephalus. Fetal dilated/enlarged lateral ventricles measuring $31 \mathrm{~mm}$. Ventriculomegaly often measures $>10 \mathrm{~mm}$ and is asymmetrical. Hydrocephalus is severe ventriculomegaly measuring $>15 \mathrm{~mm}$.

\section{Laboratory diagnosis}

If thrombocytopenia is suspected in a newborn, a complete blood count should be obtained to document whether there is isolated thrombocytopenia or if the thrombocytopenia is part of a pancytopenia syndrome. A maternal complete blood count should also be performed to exclude low maternal platelets, which is suggestive of an autoimmune thrombocytopenia. Platelet immunological tests should then be performed on parental blood and sent to one of two reference laboratories, The Blood Center of Southeastern Wisconsin (https://www.bcw.edu/bcw/index.htm) or Puget Sound Blood Center (http://www.psbc.org/home/index.htm), to confirm the diagnosis of NAIT. Antigen assays are performed on the maternal and paternal blood. ${ }^{35,36}$ If there is evidence of incompatibility, the maternal blood is processed to see if the mother has antibodies specific to that particular putative 
antigen. If maternal antibodies are present, but not specific to that antigen, HLA typing will be performed, which can show up in a nonspecific antibody screen and confound the diagnosis. If the parental genotypes are different and the mother has specific antibodies to the putative antigen, then the pregnancy is at risk for NAIT and fetal/neonatal antigen typing would then be indicated. The diagnosis of NAIT is clear when there is a maternal alloantibody directed against a paternally derived HPA antigen in the newborn that is lacking in the mother. On the other hand, NAIT is unlikely if there is no parental HPA incompatibility and antiplatelet antibodies are absent. ${ }^{37}$ If there is an initial negative maternal alloantibody screen and the suspicion of NAIT in the neonate continues to be high, it is recommended that follow-up testing for maternal antibodies be repeated 6-9 weeks postpartum as antibodies may first appear at this time. ${ }^{38}$

The two techniques that are used most often to confirm these findings are the platelet suspension immunofluorescence test (PSIFT) and the monoclonal antibody-specific immobilization of platelet antigens assay. Using PSIFT, maternal platelets are incubated with control anti-HPA-1a serum allowing antigen-antibody complex formation. These complexes are then labeled with antihuman globulin labeled with a fluorochrome, which can be detected by flow cytometry. Monoclonal antibody-specific immobilization of platelet antigens assay is a two-stage, enzyme-linked immunosorbent assay and is the confirmatory test following PSIFT. ${ }^{39}$ Monoclonal antibody-specific immobilization of platelet antigens assay is more specific but less sensitive than the PSIFT alloantibody screen but is effective in detecting antibodies to HPA-5 and HPA-15, which have fewer glycoprotein copies per platelet than HPA- $1{ }^{6}$

More recently, noninvasive screening using real-time polymerase chain reaction to detect cell-free fetal DNA isolated from maternal blood has been used to screen patients at risk; however, this is still experimental.

Unlike in Rh alloimmunization where antibody titers can be used to dictate management, antiplatelet antibody titers are not predictive of disease severity in the fetus or neonate and should not be used to monitor disease. ${ }^{40}$

\section{Screening and prevention}

Currently, it is not feasible to screen for thrombocytopenia solely by maternal ultrasound. Generally, if NAIT is diagnosed in the first pregnancy, it is usually after ultrasound findings are positive for ICH or after delivery, when an infant presents with petechiae and a low platelet count. Once there is evidence of ICH, permanent impairment has likely already occurred.
The only intervention is to prevent or decrease the risk of subsequent pregnancies from suffering the same fate. As a result, many have argued for a routine screening test for NAIT that could be performed for all pregnancies. ${ }^{41}$ A good screening test is one that attempts to screen for a clinically important and prevalent condition for which there is a treatment at an acceptable cost in relation to medical expenditure as a whole for the disease, which results in high reproducibility and is safe and acceptable to the population.

Currently, routine screening of all pregnant women for HPA-1a discordancy is not recommended, as screening is not cost-effective and the optimal treatment approach is not widely defined. ${ }^{42}$ Approximately $80 \%$ of NAIT is due to a single antigen; however, the remaining $20 \%$ is caused by multiple other antigens, some, of which are very rare. Additionally, the maternal immune response is influenced by other factors and only a minority of infants at risk will develop thrombocytopenia that is significant enough to cause ICH. There are many false negatives and positives with testing, and no adequate risk/benefit ratio or cost-effectiveness analysis has been performed. ${ }^{6,14,27,43}$

In one study, HPA-1a alloimmunization occurred in $9 \%$ of at-risk pregnancies (ie, pregnancies with a maternal HPA-1b/1b genotype and a paternal genotype either homozygous or heterozygous for HPA-1a in the presence of maternal HPA-1a antibodies). NAIT was found in 31\% of these immunized pregnancies, with perinatal ICH occurring in $10 \%$ of the pregnancies affected by NAIT. From this data, it was found that screening for HPA-1a alloimmunization only detects approximately two cases of NAIT for every 1,000 unaffected pregnancies. ${ }^{14}$ Because there is no consensus regarding the utility of screening for alloimmune antiplatelet antibodies, active management of NAIT is currently reserved for pregnancies that have already had a previously affected fetus or neonate.

\section{Recurrence risk}

In order to determine the probability of a fetus or neonate being affected in a subsequent pregnancy, parental genotyping is done through polymerase chain reaction of DNA obtained from maternal and paternal peripheral blood. If the father is heterozygous, the fetus has a $50 \%$ chance of being affected and $100 \%$ chance if the father is homozygous for the putative HPA antigen. Once the couple is determined to be at risk for NAIT in subsequent pregnancies, fetal cells obtained from chorionic villous sampling, amniocentesis, or percutaneous umbilical blood sampling (PUBS) can be used to determine if the fetus inherited the putative paternal 
antigen that the mother lacks. Amniocentesis is the preferred method because with chorionic villus sampling there is a theoretical risk that placental sampling may exacerbate maternal sensitization. PUBS carries a baseline greater risk of preterm delivery and fetal demise than amniocentesis, $1 \%$ per PUBS procedure versus $<1 \%$ after amniocentesis. Additionally, because of potentially severe thrombocytopenia, the risk of fetal hemorrhage or exsanguination is greater when FBS is performed for NAIT than for other conditions such as $\mathrm{Rh}$ alloimmunization. ${ }^{44,45}$ In an affected pregnancy, the mother is always antibody positive specific to the putative paternal and fetal platelet antigen and antigen negative to that same antigen. As such, if a fetus/neonate is diagnosed with NAIT, the couple has an $85 \%$ chance of recurrence in each subsequent pregnancy. ${ }^{46}$

\section{Prenatal management} First affected pregnancy

Unlike Rh disease where the first pregnancy is not affected, up to $40 \%-60 \%$ of infants with NAIT will be first borns. Classically, the infant is born at full-term with a normal birth weight. Thrombocytopenia is generally unexpected and oftentimes severe. One series reports a median platelet count of $19,000 / \mu \mathrm{L}$ on the 1 st day of life. Therefore, NAIT should be suspected in a thrombocytopenic neonate with extensive petechiae or visceral hemorrhage. All other possible etiologies must be ruled out including sepsis, maternal idiopathic thrombocytopenic purpura, viral exposure, and disseminated intravascular coagulation (Table 3).

If thrombocytopenia is seen in the neonate, first repeat the venous blood sample and examine the peripheral blood smear. A thorough maternal history should be obtained including medication history, history of idiopathic thrombocytopenic purpura, as well as other collagen vascular disorders. Maternal platelet count, toxoplasma gondii, other viruses (HIV, measles, and so on), rubella , cytomegalovirus, and herpes simplex (TORCH) titers, and coagulation profiles should be obtained. Parental blood should be collected for antigen typing, and the maternal serum should be tested for the presence of platelet-specific HLA alloantibodies. If the suspicion for NAIT is high, treatment should not be withheld until the laboratories return given the high likelihood of continued bleeding. Neonatal head ultrasounds should also be performed to rule out any evidence of ICH.

\section{Antepartum management in subsequent pregnancies}

Once NAIT has been diagnosed in a previous pregnancy, it is known that all subsequent pregnancies are at risk for severe disease. ${ }^{33}$ The goal is to initiate treatment early in order to prevent the devastating and potentially life-threatening manifestations of disease. In light of the wide disease spectrum, treatment should be tailored to each individual patient based on previous pregnancy history. Factors involved in treatment initiation should include the presence or absence of ICH in the previously affected pregnancy, and, if present, the timing of its occurrence. The different treatment strategies depend on predicted severity of the disease, which can be separated into three categories: standard risk, high risk and very high risk (Table 4). A standard-risk pregnancy is defined as a pregnancy where a previous sibling had isolated thrombocytopenia and no previous ICH. High-risk pregnancies are pregnancies where a previous sibling suffered an ICH at $\geq 28$ weeks or after birth, and very high risk pregnancies are those where a previous pregnancy resulted in $\mathrm{ICH}$ at $<28$ weeks or was complicated by an intrauterine fetal demise.

Because ICH is one of the most devastating antenatal complications of NAIT, the goal is to prevent its occurrence once a diagnosis of NAIT has been made. Studies have demonstrated that the risk of $\mathrm{ICH}$ is rare if platelets are kept above $20,000 / \mu \mathrm{L}$; therefore, the goal of treatment is to keep platelet levels above that threshold. IgG alloantibodies can cross the placenta as early as 14 weeks gestation and fetal glycoproteins are expressed on platelets starting at 16 weeks; therefore, symptoms of NAIT can present as early as 16 weeks. ${ }^{23,42,47,48}$ For this reason, treatment should be initiated early, especially in those at high risk and very high risk for severe disease.

Because the blood tests are sent out to reference laboratories and it can take several weeks to confirm the diagnosis, if NAIT is suspected, treatment should be initiated before obtaining laboratory confirmation of disease.

Table 4 Approach to risk assessment for women with a previous maternal history of NAIT

\begin{tabular}{ll}
\hline Clinical features of prior pregnancy & $\begin{array}{l}\text { Risk of neonate } \\
\text { with severe NAIT }\end{array}$ \\
\hline $\begin{array}{l}\text { Previous sibling with isolated thrombocytopenia, } \\
\text { no ICH }\end{array}$ & Standard risk \\
$\begin{array}{l}\text { Previous sibling with antenatal } \mathrm{ICH} \geq 28 \text { weeks } \\
\text { Previous sibling with peripartum } \mathrm{ICH}\end{array}$ & High risk \\
Previous sibling with antenatal $\mathrm{ICH}<28$ weeks & High risk \\
Previous sibling with in utero fetal death & Very high risk \\
\hline
\end{tabular}

Notes: Criteria for diagnosis include a clinical history consistent with NAIT. Adapted with permission from: A review of pathophysiology and current treatment for neonatal alloimmune thrombocytopenia (NAIT) and introducing the Australian NAIT registry. McQuilten ZK, Wood EM, Savoia H, et al. Aust N Z J Obstet Gynaecol, 51. Copyright $\odot 2011$ John Wiley and Sons. ${ }^{64}$

Abbreviations: $\mathrm{ICH}$, intracranial hemorrhage; NAIT, neonatal alloimmune thrombocytopenia. 
A multidisciplinary approach to care should be undertaken involving a maternal-fetal medicine specialist, neonatologist, hematologist, and the blood bank. Affected pregnancies should also be monitored with serial growth ultrasounds every 2-3 weeks and fetal magnetic resonance imaging, if available, every 4-6 weeks to assess for evidence of ICH.

Treatment options include maternal intravenous immunoglobulin (IVIG), corticosteroid administration, FBS to monitor platelet counts with platelet transfusions as needed, in utero IVIG administration, and early delivery (after 32 weeks gestation). More recent literature has shown that noninvasive treatment with IVIG and corticosteroids is safe, effective, and preferred over the more invasive serial PUBS and transfusions secondary to the procedure-related complications including fetal loss or miscarriage. ${ }^{18,49,50}$ Data from previously published studies recommend that treatment should be initiated antenatally in order to raise the fetal platelet count thereby preventing in utero bleeding. ${ }^{32,51}$

\section{IVIG and prednisone}

IVIG is a blood product administered intravenously that contains pooled, polyvalent, and IgG antibodies extracted from the plasma of over 1,000 blood donors. It is costly, resulting in at least US $\$ 1,000$ per dose usually given as a weekly infusion over a period of 6-12 hours. When the IVIG dose is $2 \mathrm{~g} / \mathrm{kg} / \mathrm{wk}$, it can be infused as doses of $1 \mathrm{~g} / \mathrm{kg}$ twice a week. Compared to no treatment, prospective studies have shown IVIG to be more effective in reducing the risk of ICH. One study showed that only $2.7 \%$ of fetuses treated with IVIG developed ICH. ${ }^{52}$ The only way to monitor efficacy of IVIG is to perform FBS. A randomized controlled trial found that $75 \%$ of pregnancies respond to weekly IVIG, with half of the nonresponders improving after the addition of high-dose prednisone $(1 \mathrm{mg} / \mathrm{kg} / \mathrm{d}){ }^{53}$ However, treatment with both IVIG and steroids are not without maternal and fetal risks. IVIG can be associated with a local reaction at the injection site, fevers, rash, headache, flushing, myalgia, and chills. There is also a small increased risk of hepatitis and HIV. More severe reactions include aseptic meningitis, acute kidney injury, arterial or venous thrombosis, hemolytic anemia, and stroke. Prior to undergoing IVIG treatment, the patient should be premedicated with acetaminophen $650 \mathrm{mg}$ and diphenhydramine $25-50 \mathrm{mg}$. By infusing slowly, side effects can be minimized. Chronic steroid use can lead to osteoporosis, impaired glucose tolerance and gestational diabetes, hypertension, immunosuppression, mood swings, and fetal structural anomalies such as midline facial defects. Those on chronic steroids should be screened for gestational diabetes and receive calcium supplementation $(1 \mathrm{~g} / \mathrm{d})$ and vitamin $\mathrm{D}$ $(400 \mathrm{u} / \mathrm{d}) .^{54}$

IVIG has an unclear mechanism of action; however, it is thought to work by diluting the anti-HPA antibodies in the maternal circulation resulting in a lower proportion of antiHPA antibodies reaching the Fc-receptors on the placenta. IVIG is thought to block the placental Fc-receptors thereby decreasing the placental transmission of maternal antibodies across the placenta. IVIG may also block the Fc-receptors on macrophages in the fetal circulation and prohibit the destruction of antibody-covered cells. Finally, IVIG is thought to suppress maternal IgG antibody production. ${ }^{18}$ The fetal risks after maternal transfusion with IVIG are minimal. Follow-up studies after antenatal treatment with IVIG have not been shown to have adverse effects on the general health or neurodevelopmental outcome in the affected fetus with one study showing improved neurodevelopmental outcomes despite delivery at an earlier gestational age. ${ }^{55,56}$

\section{Percutaneous umbilical blood sampling}

Another treatment option to consider is PUBS, also referred to as cordocentesis or FBS. More recently, management with serial PUBS has fallen out of favor because of the fetal and maternal risks from repeated invasive procedures when effective noninvasive alternatives are available. Currently, the main indications for performing PUBS procedures are to quantify platelet levels, to determine if noninvasive treatment measures have been effective or adjustments with therapy need to be made, to determine mode of delivery, and if necessary, for platelet transfusion.

PUBS should be performed close to an operating room, either on labor and delivery or in an ultrasound unit in close proximity to the operating room, should procedural complications arise and an emergency cesarean section is required. The procedure is usually performed starting at 20 weeks, and once viability is reached ( 24 weeks), it is recommended that the patient receive a course of corticosteroids (either betamethasone or dexamethasone) 24-48 hours prior to the procedure in order to stimulate fetal lung maturity in case of delivery. Patients are usually instructed to avoid oral intake for 6-8 hours preprocedure and will have intravenous access secured on presentation. Before starting, the abdomen is prepped and draped in a sterile fashion using chlorhexidine. With the assistance of ultrasound, fetal presentation is identified, a preprocedure fetal heart rate is obtained, and the placental cord insertion site is located. Lidocaine $1 \%$ can be administered in the subcutaneous layer if preferred by the patient. A 22-gauge spinal needle is typically used and, under 
ultrasound guidance, inserted into the umbilical vein at the placental cord insertion site. A 22-gauge needle as opposed to a 20-gauge needle is used to minimize the risk of bleeding from the puncture site as studies have found a two fold increased risk of fetal exsanguination after PUBS for NAIT compared to PUBS for other indications. ${ }^{17}$

After needle insertion, a fetal blood sample is withdrawn in a 1-cc heparinized syringe to identify the fetal platelet count. Additionally, a mean corpuscular red cell volume is assessed to confirm that the blood sample is fetal as opposed to maternal. A mean corpuscular volume $>100 \mu \mathrm{L}$ suggests fetal red cells.

After a blood sample is obtained and confirmed to be fetal, a short-acting paralytic such as atracurium $(0.4 \mathrm{mg}$ / $\mathrm{kg})$ or vecuronium $(0.1 \mathrm{mg} / \mathrm{kg})$ can be administered to minimize fetal movement. ${ }^{57}$ Platelets should only be transfused if there is evidence of thrombocytopenia. If platelets are given empirically and the fetal platelet count is normal, the fetus is at increased risk for thrombocytosis, hypercoagulability, and intrauterine demise. Transfusion of platelets via PUBS is usually indicated when the platelet count is less than $50,000 / \mu \mathrm{L}$ with the goal transfusion platelet count to be above $50,000 / \mu \mathrm{L}$ to allow for clotting from the umbilical cord after transfusion and needle removal. If transfusion is indicated, transfusion with maternal platelets is best. Maternal platelets can be obtained by apheresis at least 24-48 hours before the procedure. The platelets must be washed in order to remove the maternal alloantibodies and gamma irradiated to avoid graft versus host disease. If maternal platelets are not available, platelets can be obtained from a national registry of HPA-typed donors. ${ }^{28}$

If platelets are transfused, the primary operator should visualize the transfused platelets streaming away from the needle as they mix with fetal blood in the umbilical vein throughout the procedure. Care should be taken to intermittently assess the fetal heart rate for bradycardia. Once the platelet transfusion is completed, flush the needle with normal saline, withdraw, and discard $1 \mathrm{cc}$ of blood. Next, in a heparinized 1-cc syringe, fill the syringe to obtain a postprocedure platelet count in order to determine the final platelet count before needle removal. In some cases, if transfusion is not performed, a small aliquot of $3-5 \mathrm{~mL}$ of platelets can be infused to decrease the risk of exsanguination. The puncture site should be visualized upon completion of the procedure to determine if there is excessive bleeding or streaming. If $>24$ weeks gestation, the patient should be monitored on the labor floor for up to 4 hours postprocedure in order to ensure a reassuring fetal status.
The required transfusion volume can be obtained using the following equation:

$$
\begin{aligned}
& \text { Volume transfused }(\mathrm{mL}) \\
& \text { Estimated fetoplacental volume }(\mathrm{mL})^{*} \\
& =\frac{\times(\text { Target final }- \text { Initial platelet count }) \times 2}{\text { Platelet count of transfused concentrate }}
\end{aligned}
$$

*The fetoplacental volume is calculated by multiplying the ultrasound-estimated fetal weight in grams by 0.14 . In order to allow for possible platelet sequestration in the fetal spleen or liver, the numerator is multiplied by a factor of 2 .

Platelet counts fall by approximately 10,000-20,000/ $\mu \mathrm{L}$ each week. Therefore, because the half-life of transfused platelets is about 3 days, a fetus should be transfused to a target platelet count of $300,000-500,000 / \mu \mathrm{L}$ to achieve a nadir platelet count of $30,000-50,000 / \mu \mathrm{L}$ at the time of the next transfusion. ${ }^{58}$ Once transfusions are initiated, they are usually repeated weekly because of the limited half-life of transfused platelets. There is currently no consensus regarding gestational age of delivery after serial platelet transfusions; however, delivery after 32 weeks of gestation may be appropriate when weighing the risks of prematurity to the risks of continued transfusions and possible worsening NAIT.

PUBS and in utero transfusion is associated with significant procedure-related risks including fetal bradycardia requiring delivery, exsanguination, cord hematoma or occlusion, and death. A study by Overton et al identified 12 pregnancies that underwent a total of 84 transfusions. They found that the risk of pregnancy loss from each PUBS and transfusion is $1 \%$, with a cumulative loss rate as high as $8 \%$ per pregnancy. ${ }^{44}$

\section{Antepartum management based on severity of disease}

\section{Standard risk group}

Pregnancies with a history of a previous fetus or neonate with thrombocytopenia, serologically confirmed NAIT, without evidence of $\mathrm{ICH}$ are classified as standard risk. Published studies recommend treatment with IVIG starting at 20 weeks at a dose of $2 \mathrm{~g} / \mathrm{kg} / \mathrm{wk}$ alone or $1 \mathrm{~g} / \mathrm{kg} /$ wk plus prednisone $0.5 \mathrm{mg} / \mathrm{kg} / \mathrm{d}$ as both treatment options are comparable. ${ }^{24,44,59}$ FBS is then recommended at 32 weeks to determine the fetal platelet count and in order to decide if salvage therapy is necessary. Salvage therapy should be implemented if the fetal platelet counts is $<30-50,000 / \mu \mathrm{L}$ by adding prednisone $0.5 \mathrm{mg} / \mathrm{kg} / \mathrm{d}$ to the IVIG regimen only or increasing the IVIG 
dosing to $2 \mathrm{~g} / \mathrm{kg} / \mathrm{wk}$ if previously receiving combination therapy of IVIG $1 \mathrm{mg} / \mathrm{kg} / \mathrm{wk}$ and prednisone $0.5 \mathrm{mg} / \mathrm{kg} / \mathrm{d}$. A salvage failure is considered a birth platelet count less than $30,000 / \mu \mathrm{L}$ (Figure 2). The decision for attempted vaginal delivery should be discussed in detail with the understanding that a vaginal delivery is only recommended for those whose fetal platelets are $\geq 100,000 / \mu \mathrm{L}$ at 32 weeks and remain compliant with therapy. An alternative approach is to start salvage therapy on everyone at 32 weeks without knowledge of platelet status followed by FBS at 36 weeks in order to determine the mode of delivery. A randomized controlled trial by Berkowitz et al demonstrated that 0 of 73 patients suffered an ICH in the antepartum period after using this treatment regimen. ${ }^{59}$

\section{High-risk group}

Those patients at high risk for severe disease are patients who are pregnant with serologically confirmed NAIT with a previous fetus or newborn affected by NAIT who had an ICH at $\geq 28$ weeks or in the neonatal period. According to a study by Bussel et al, treatment is recommended starting at 12 weeks with IVIG $1 \mathrm{mg} / \mathrm{kg} / \mathrm{wk}$. At 20 weeks, it is recommended to empirically add prednisone $1 \mathrm{mg} / \mathrm{kg} / \mathrm{d}$ or to increase the IVIG dose to $2 \mathrm{~g} / \mathrm{kg} / \mathrm{wk}$. As an alternative, FBS can be performed at 32 weeks to determine if salvage therapy needs to be initiated. If the platelet count is $<30,000 / \mu \mathrm{L}$, or FBS is not possible, salvage therapy is initiated by adding prednisone $1 \mathrm{mg} / \mathrm{kg} / \mathrm{d}$ to those not previously on it or by increasing the IVIG dose to $2 \mathrm{~g} / \mathrm{kg} / \mathrm{wk}$. Delivery after 32 weeks is also an option if indicated..$^{40}$

\section{Very high risk group}

If the patient is at extremely high risk for severe disease that being serologically confirmed NAIT and previous fetus with an $\mathrm{ICH}$ at $<28$ weeks or history of an intrauterine fetal demise, aggressive therapy should start at 12 weeks with $2 \mathrm{~g} / \mathrm{kg} /$ wk of IVIG. All patients should be started on $1 \mathrm{mg} / \mathrm{kg} /$ wk of prednisone at 20 weeks for the remainder of the pregnancy. FBS should be performed at 32 weeks to see if additional salvage therapy, fetal transfusions, or delivery is needed. In the study by Bussel et al, all seven extremely high risk patients treated with this regimen delivered infants with birth platelet counts of $>50,000 / \mu \mathrm{L}$, and none suffered an $\mathrm{ICH} .{ }^{40}$

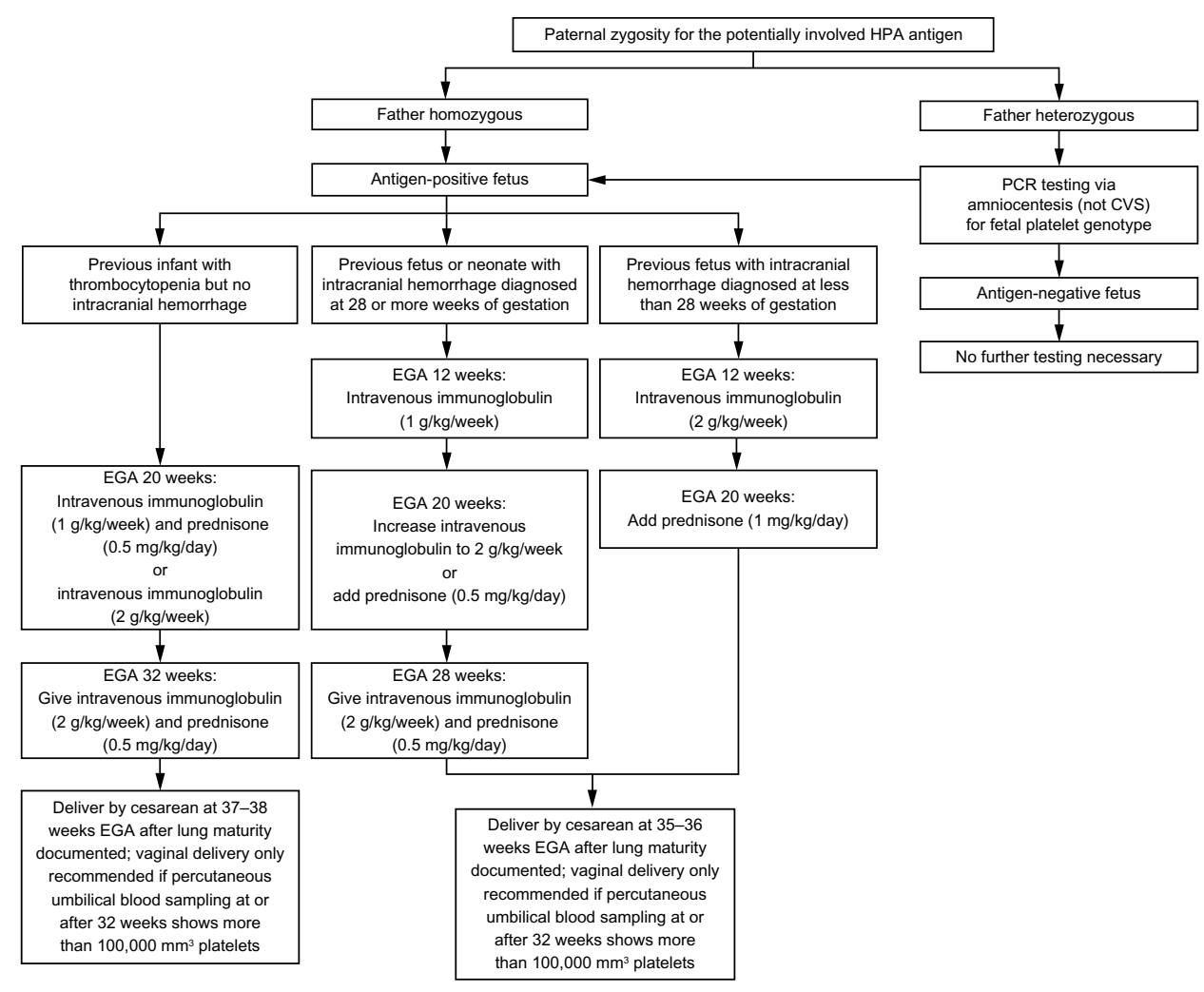

Figure 2 Platelet alloimmunization algorithm.

Note: Reproduced with permission from: Pacheco LD, Berkowitz RL, Moise KJ, et al. Fetal and neonatal alloimmune thrombocytopenia. Obstet and Gynecol. 20 I ; I I8: I I571163.54

Abbreviations: CVS, chorionic villus sampling; EGA, estimated gestational age; HPA, human platelet antigen; PCR, polymerase chain reaction. 
For patients who do not respond to IVIG and glucocorticoid administration, an alternative treatment includes serial-matched platelet transfusion. Platelet transfusions only transiently increase the fetal platelet count because transfused platelets are also targeted by maternal antibodies. ${ }^{4}$ All treatment regimens should be continued until delivery. Although the data are conflicting regarding the optimal mode of delivery, early delivery via cesarean section may help reduce the risk of ICH in severely thrombocytopenic fetuses.

\section{Mode of delivery}

Timing of delivery is variable with delivery for severe disease recommended at an earlier gestational age. Currently, there is no absolute recommendation for gestational age of delivery or preferred mode of delivery. There is no definitive evidence that cesarean delivery prevents $\mathrm{ICH} .{ }^{18}$ Most studies suggest liberal use of cesarean delivery for those at high risk or very high risk for disease with consideration of vaginal birth only if the platelet count is greater than $50,000-100,000 / \mu \mathrm{L}$ prior to delivery. For those at standard risk, vaginal deliveries are thought to be safe. A prospective study from the Netherlands found that vaginal delivery is safe in pregnancies affected by NAIT when there was no ICH detected in the previous pregnancy. In this study, 23 women delivered vaginally and nine had cesarean deliveries, all for obstetric indications. Severe neonatal thrombocytopenia $(<50,000 / \mu \mathrm{L})$ was seen in four deliveries, of which three were born vaginally. None of the neonates had signs of ICH or bleeding after delivery. ${ }^{60}$

For those in the higher risk groups who desire vaginal delivery, FBS is recommended at 32 weeks and if the platelet count is greater than $100,000 / \mu \mathrm{L}$, the patient should continue therapy and then may undergo induction at 37-38 weeks. If the patient decides to forego PUBS FBS at 32 weeks, but desires a vaginal delivery, PUBS FBS can be performed between 36 and 37 weeks and if the fetal platelet count is above $50,000 / \mu \mathrm{L}$, a vaginal delivery can be offered. ${ }^{59}$

If PUBS FBS is not performed, delivery after documented fetal lung maturity by cesarean section is recommended at $37-38$ weeks if there is no history of ICH in a prior pregnancy and at 35-36 weeks in those with a prior affected newborn or fetus with an ICH. For those in the very high risk group, cesarean delivery is usually recommended regardless of platelet count. Care should be taken to avoid fetal trauma with vacuum, forceps, fetal scalp electrodes, and external cephalic version in all cases affected by NAIT. ${ }^{54}$

Although up to $10 \%$ of women can have gestational thrombocytopenia, maternal platelets are usually normal and there should be no contraindications to regional anesthesia.
In all cases, delivery should occur at a tertiary care center secondary to the risk for ICH and need for platelet transfusion after delivery.

\section{Neonatal management}

Although the thrombocytopenia caused by NAIT usually resolves spontaneously within 1-2 weeks after delivery, serious bleeding, including ICH, can still occur in the immediate postpartum period. Serial neonatal platelet counts should be obtained for the first 5-7 days after delivery. ${ }^{18,61,62}$ The goal of therapy in the first 72-96 hours of life is to keep platelet levels above $30,000 / \mu \mathrm{L}$ without active bleeding and above $50,000-100,000 / \mu \mathrm{L}$ if there is evidence of active bleeding. ${ }^{24,27,28}$ Neonates can be treated similarly to mothers in the antenatal period with IVIG, steroids, or platelet transfusions if rapid correction of the platelet count is indicated. Maternal or donor platelets should be available for transfusion after delivery. The recommended transfusion is $10 \mathrm{~mL}$ of platelets per kilogram of neonatal weight where $1 \mathrm{~mL}$ of platelet increases the platelet count by $5,000 / \mu \mathrm{L}$.

If a platelet transfusion is necessary, washed and irradiated maternal platelets or HPA-compatible donor platelets obtained from a regional registry of HPA-typed donors are recommended. Of note, maternal platelets are no longer used as frequently because the process of washing and irradiating maternal platelets to make them suitable for transfusion is lengthy and can reduce platelet function and is therefore not the best option when an emergent transfusion is needed. If postpartum transfusion cannot be delayed, it is safe and beneficial for the neonate to receive a random platelet transfusion with or without IVIG. ${ }^{28,62,63}$ One retrospective study showed that transfusion of random-donor platelets alone was effective in correcting critically low platelet counts and preventing further bleeding.

\section{Conclusion}

It is important to remember that although NAIT is a rare condition, however, it is the most common cause of severe thrombocytopenia in the "well" term neonate. The disease spectrum may vary from mild to severe thrombocytopenia with ICH and possibly death. Any term neonate with no identifiable cause for thrombocytopenia should have parental samples sent for NAIT testing. Treatment in all subsequent pregnancies should be initiated early and should include IVIG with or without the addition of steroids, if necessary. If fetal transfusion is required, HPA-compatible donor platelets are preferred; however, random-donor platelet transfusions have been shown to be a safe, effective, and an immediate 
way to increase the platelet count if HPA-matched platelets are unavailable. A multidisciplinary approach should be used to care for women with pregnancies affected by NAIT including maternal-fetal medicine specialists, hematologists, neonatologists, and the blood bank, with delivery occurring at a tertiary health care center.

\section{Disclosure}

The authors report no conflicts of interest in this work.

\section{References}

1. Sainio S, Järvenpää AL, Renlund M, Riikonen S, Teramo K, Kekomäki R. Thrombocytopenia in term infants: a population-based study. Obstet Gynecol. 2000;95:441-446.

2. Kamphuis MM, Paridaans NP, Porcelijn L, Lopriore E, Oepkes D. Incidence and consequences of neonatal alloimmune thrombocytopenia: a systematic review. Pediatrics. 2014;133:715-721.

3. Bussel JB, Sola-Visner M. Current approaches to the evaluation and management of the fetus and neonate with immune thrombocytopenia Semin Perinatol. 2009;33:35-42.

4. Letsky EA, Greaves M. Guidelines on the investigation and management of thrombocytopenia in pregnancy and neonatal alloimmune thrombocytopenia. Maternal and Neonatal Haemostasis Working Party of the Haemostasis and Thrombosis Task Force of the British Society for Haematology. Br J Haematol. 1996;95:21-26.

5. Curtis BR, McFarland JG. Detection and identification of platelet antibodies and antigens in the clinical laboratory. Immunohematology. 2009;25:125-135.

6. Risson DC, Davies MW, Williams BA. Review of neonatal alloimmune thrombocytopenia. J Paediatr Child Health. 2012;48:816-822.

7. Metcalfe P, Watkins NA, Ouwehand WH, et al. Nomenclature of human platelet antigens. Vox Sang. 2003;85:240-245.

8. Davoren A, Curtis BR, Aster RH, McFarland JG. Human platelet antigen-specific alloantibodies implicated in 1162 cases of neonata alloimmune thrombocytopenia. Transfusion. 2004;44:1220-1225.

9. Seo DH, Park SS, Kim DW, Furihata K, Ueno I, Han KS. Gene frequencies of eight human platelet-specific antigens in Koreans. Transfus Med. 1998;8:129-132.

10. Tanaka S, Ohnoki S, Shibata H, Okubo Y, Yamaguchi H, Shibata Y. Gene frequencies of human platelet antigens on glycoprotein IIIa in Japanese. Transfusion. 1996;36:813-817.

11. Kupatawintu P, Nathalang O, O-Charoen R, Patmasiriwat P. Gene frequencies of the HPA-1 to 6 and Gov human platelet antigens in Thai blood donors. Immunohematology. 2005;21:5-9.

12. Kekomaki S, Partanen J, Kekomaki R. Platelet alloantigens HPA-1, -2, $-3,-5$ and $-6 b$ in Finns. Transfus Med. 1995;5:193-198.

13. Kim HO, Jin Y, Kickler TS, Blakemore K, Kwon OH, Bray PF. Gene frequencies of the five major human platelet antigens in African American, white, and Korean populations. Transfusion. 1995;35 863-867.

14. Kamphuis MM, Paridaans N, Porcelijn L, et al. Screening in pregnancy for fetal or neonatal alloimmune thrombocytopenia: systematic review. Int J Obstetrics Gynaecol. 2010;117:1335-1343.

15. Davoren A, McParland P, Crowley J, Barnes A, Kelly G, Murphy WG. Antenatal screening for human platelet antigen-1a: results of a prospective study at a large maternity hospital in Ireland. Int J Obstet Gynaecol. 2003;110:492-496.

16. Semple JW. Processed platelet HPA1a peptides au naturel. Blood. 2009;114:1724-1725.

17. Radder CM, Brand A, Kanhai HH. Will it ever be possible to balance the risk of intracranial haemorrhage in fetal or neonatal alloimmune thrombocytopenia against the risk of treatment strategies to prevent it? Vox Sang. 2003;84:318-325.
18. Kamphuis MM, Oepkes D. Fetal and neonatal alloimmune thrombocytopenia: prenatal interventions. Prenat Diagn. 2011;31:712-719.

19. Williamson LM, Hackett G, Rennie J, et al. The natural history of fetomaternal alloimmunization to the platelet-specific antigen HPA-1a (PlA1, Zwa) as determined by antenatal screening. Blood. 1998;92: 2280-2287.

20. Strong NK, Eddleman KA. Diagnosis and management of neonatal alloimmune thrombocytopenia in pregnancy. Clin Lab Med. 2013;33: 311-325.

21. Bonacossa IA, Jocelyn LJ. Alloimmune thrombocytopenia of the newborn: neurodevelopmental sequelae. Am J Perinatol. 1996;13: 211-215.

22. Herman JH, Jumbelic MI, Ancona RJ, Kickler TS. In utero cerebral hemorrhage in alloimmune thrombocytopenia. Am J Pediatr Hematol Oncol. 1986;8:312-317.

23. Mueller-Eckhardt C, Kiefel V, Grubert A, et al. 348 cases of suspected neonatal alloimmune thrombocytopenia. Lancet. 1989;1:363-366.

24. Van Der Lugt NM, Kamphuis MM, Paridaans NP, et al. Neonatal outcome in alloimmune thrombocytopenia after maternal treatment with intravenous immunoglobulin. Blood Transfus. 2014; $12: 1-6$.

25. Bussel JB, Zacharoulis S, Kramer K, McFarland JG, Pauliny J, Kaplan C. Clinical and diagnostic comparison of neonatal alloimmune thrombocytopenia to non-immune cases of thrombocytopenia. Pediatric Blood Cancer. 2005;45:176-183.

26. Spencer JA, Burrows RF. Feto-maternal alloimmune thrombocytopenia: a literature review and statistical analysis. Aus NZJ Obstet Gynaecol. 2001;41:45-55.

27. Espinoza JP, Caradeux J, Norwitz ER, Illanes SE. Fetal and neonatal alloimmune thrombocytopenia. Rev Obstet Gynecol. 2013;6: e15-e21.

28. Bertrand G, Kaplan C. How do we treat fetal and neonatal alloimmune thrombocytopenia? Transfusion. 2014;54:1698-1703.

29. Bussel J. Diagnosis and management of the fetus and neonate with alloimmune thrombocytopenia. J Thromb Haemostasis. 2009; 7(Suppl 1):253-257.

30. Ghevaert C, Campbell K, Walton J, et al. Management and outcome of 200 cases of fetomaternal alloimmune thrombocytopenia. Transfusion. 2007;47:901-910.

31. Killie MK, Husebekk A, Kjeldsen-Kragh J, Skogen B. A prospective study of maternal anti-HPA 1a antibody level as a potential predictor of alloimmune thrombocytopenia in the newborn. Haematologica. 2008;93:870-877.

32. Bussel JB, Zabusky MR, Berkowitz RL, McFarland JG. Fetal alloimmune thrombocytopenia. N Engl J Med. 1997;337:22-26.

33. Birchall JE, Murphy MF, Kaplan C, Kroll H. European collaborative study of the antenatal management of feto-maternal alloimmune thrombocytopenia. Br J Haematol. 2003;122:275-288.

34. Bussel J, Kaplan C. The fetal and neonatal consequences of maternal alloimmune thrombocytopenia. Bailliere Clin Haematol. 1998;11: 391-408.

35. Curtis BR. Genotyping for human platelet alloantigen polymorphisms: applications in the diagnosis of alloimmune platelet disorders. Seminars Thromb Hemostasis. 2008;34:539-548.

36. Hurd CM, Cavanagh G, Schuh A, Ouwehand WH, Metcalfe P. Genotyping for platelet-specific antigens: techniques for the detection of single nucleotide polymorphisms. Vox Sang. 2002; 83:1-12.

37. Blanchette VS, Chen L, de Friedberg ZS, Hogan VA, Trudel E, Décary F. Alloimmunization to the PlA1 platelet antigen: results of a prospective study. Br J Haematol. 1990;74:209-215.

38. Dawkins B. Monitoring anti-HPA-1a platelet antibody levels during pregnancy using the MAIPA test. Vox Sang. 1995;68:27-34.

39. Kiefel V, Santoso S, Weisheit M, Mueller-Eckhardt C. Monoclonal antibody - specific immobilization of platelet antigens (MAIPA): a new tool for the identification of platelet-reactive antibodies. Blood. 1987;70:1722-1726. 
40. Bussel JB, Berkowitz RL, Hung C, et al. Intracranial hemorrhage in alloimmune thrombocytopenia: stratified management to prevent recurrence in the subsequent affected fetus. Am J Obstet Gynecol. 2010; 203(135): e131-e114.

41. Turner ML, Bessos H, Fagge T, et al. Prospective epidemiologic study of the outcome and cost-effectiveness of antenatal screening to detect neonatal alloimmune thrombocytopenia due to anti-HPA-1a. Transfusion. 2005;45:1945-1956.

42. Skogen B, Killie MK, Kjeldsen-Kragh J, et al. Reconsidering fetal and neonatal alloimmune thrombocytopenia with a focus on screening and prevention. Exp Rev Hematol. 2010;3:559-566.

43. Murphy MF, Williamson LM, Urbaniak SJ. Antenatal screening for fetomaternal alloimmune thrombocytopenia: should we be doing it? Vox Sang. 2002;83(Suppl 1):409-416.

44. Overton TG, Duncan KR, Jolly M, Letsky E, Fisk NM. Serial aggressive platelet transfusion for fetal alloimmune thrombocytopenia: platelet dynamics and perinatal outcome. Am J Obstet Gynecol. 2002;186: 826-831.

45. Paidas MJ, Berkowitz RL, Lynch L, et al. Alloimmune thrombocytopenia: fetal and neonatal losses related to cordocentesis. Am J Obstet Gynecol. 1995;172:475-479.

46. Yinon Y, Spira M, Solomon O, et al. Antenatal noninvasive treatment of patients at risk for alloimmune thrombocytopenia without a history of intracranial hemorrhage. Am J Obstet Gynecol. 2006;195:1153-1157.

47. Kaplan C. Alloimmune thrombocytopenia of the fetus and the newborn. Blood Rev. 2002;16:69-72.

48. Kaplan C. Neonatal alloimmune thrombocytopenia. Haematologica. 2008;93:805-807.

49. van den Akker ES, Oepkes D, Lopriore E, Brand A, Kanhai HH. Noninvasive antenatal management of fetal and neonatal alloimmune thrombocytopenia: safe and effective. Int J Obstet Gynaecol. 2007;114: 469-473.

50. Rayment R, Brunskill SJ, Soothill PW, Roberts DJ, Bussel JB, Murphy MF. Antenatal interventions for fetomaternal alloimmune thrombocytopenia. Cochrane Database Syst Rev. 2011;5: CD004226.

51. Berkowitz RL, Kolb EA, McFarland JG, et al. Parallel randomized trials of risk-based therapy for fetal alloimmune thrombocytopenia. Obstet Gynecol. 2006;107:91-96.

52. Vinograd CA, Bussel JB. Antenatal treatment of fetal alloimmune thrombocytopenia: a current perspective. Haematologica. 2010;95: $1807-1811$
53. Bussel JB, Berkowitz RL, Lynch L, et al. Antenatal management of alloimmune thrombocytopenia with intravenous gamma-globulin: a randomized trial of the addition of low-dose steroid to intravenous gamma-globulin. Am J Obstet Gynecol. 1996;174:1414-1423.

54. Pacheco LD, Berkowitz RL, Moise KJ Jr, Bussel JB, McFarland JG, Saade GR. Fetal and neonatal alloimmune thrombocytopenia: a management algorithm based on risk stratification. Obstet Gynecol. 2011;118: $1157-1163$

55. Radder CM, de Haan MJ, Brand A, Stoelhorst GM, Veen S, Kanhai HH. Follow up of children after antenatal treatment for alloimmune thrombocytopenia. Early Hum Dev. 2004;80:65-76.

56. Ward MJ, Pauliny J, Lipper EG, Bussel JB. Long-term effects of fetal and neonatal alloimmune thrombocytopenia and its antenatal treatment on the medical and developmental outcomes of affected children. Am J Perinatol. 2006;23:487-492.

57. Mouw RJ, Klumper F, Hermans J, Brandenburg HC, Kanhai HH. Effect of atracurium or pancuronium on the anemic fetus during and directly after intravascular intrauterine transfusion. A double blind randomized study. Acta Obstetricia Gynecologica Scandinavica. 1999;78: 763-767.

58. Murphy MF, Waters AH, Doughty HA, et al. Antenatal management of fetomaternal alloimmune thrombocytopenia - report of 15 affected pregnancies. Transfus Med. 1994;4:281-292.

59. Berkowitz RL, Lesser ML, McFarland JG, et al. Antepartum treatment without early cordocentesis for standard-risk alloimmune thrombocytopenia: a randomized controlled trial. Obstet Gynecol. 2007;110:249-255.

60. van den Akker E, Oepkes D, Brand A, Kanhai HH. Vaginal delivery for fetuses at risk of alloimmune thrombocytopenia? Int J Obstet Gynaecol. 2006;113:781-783.

61. Chakravorty S, Roberts I. How I manage neonatal thrombocytopenia. Br J Haematol. 2012;156:155-162.

62. Bakchoul T, Bassler D, Heckmann M, et al. Management of infants born with severe neonatal alloimmune thrombocytopenia: the role of platelet transfusions and intravenous immunoglobulin. Transfusion. 2014;54:640-645.

63. Kiefel V, Bassler D, Kroll H, et al. Antigen-positive platelet transfusion in neonatal alloimmune thrombocytopenia (NAIT). Blood. 2006;107: 3761-3763.

64. McQuilten ZK, Wood EM, Savoia H, et al. A review of pathophysiology and current treatment for neonatal alloimmune thrombocytopenia (NAIT) and introducing the Australian NAIT registry. Aust NZJ Obstet Gynaecol. 2011;51:191-198.
International Journal of Clinical Transfusion Medicine

\section{Publish your work in this journal}

International Journal of Clinical Transfusion Medicine is an international, peer-reviewed, open access, online journal publishing clinicalexperimental, policy-making and evidence-based practices of all topics pertaining to clinical transfusion medicine. Original research, short reports, reviews, case reports and commentaries are invited.

\section{Dovepress}

The manuscript management system is completely online and includes a very quick and fair peer-review system, which is all easy to use. Visit http://www.dovepress.com/testimonials.php to read real quotes from published authors. 